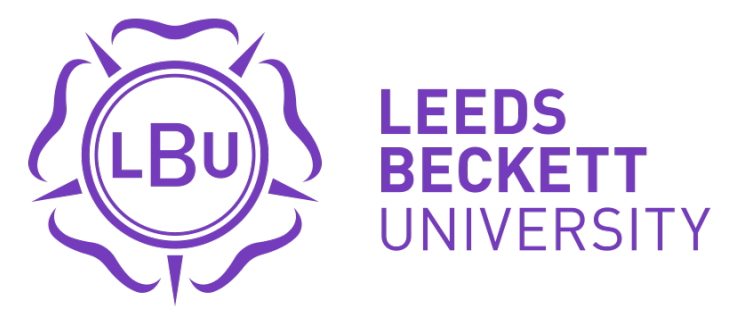

Citation:

Perepelkin, NV (2018) Non-iterative Rauscher method for 1-DOF system: a new approach to studying non-autonomous system via equivalent autonomous one. Nonlinear Dynamics, 93 (1). pp. 149-166. ISSN 0924-090X DOI: https://doi.org/10.1007/s11071-017-3841-2

Link to Leeds Beckett Repository record:

http://eprints.leedsbeckett.ac.uk/6685/

Document Version:

Article

The aim of the Leeds Beckett Repository is to provide open access to our research, as required by funder policies and permitted by publishers and copyright law.

The Leeds Beckett repository holds a wide range of publications, each of which has been checked for copyright and the relevant embargo period has been applied by the Research Services team.

We operate on a standard take-down policy. If you are the author or publisher of an output and you would like it removed from the repository, please contact us and we will investigate on a case-by-case basis.

Each thesis in the repository has been cleared where necessary by the author for third party copyright. If you would like a thesis to be removed from the repository or believe there is an issue with copyright, please contact us on openaccess@leedsbeckett.ac.uk and we will investigate on a case-by-case basis. 


\title{
Non-Iterative Rauscher Method for 1-DOF System: a New Approach to Studying Non- Autonomous System via Equivalent Autonomous One
}

\author{
Nikolay V. Perepelkin \\ National Technical University "Kharkiv Polytechnic Institute”, Kharkiv, Ukraine. \\ nickv.perepelkin@gmail.com
}

Rauscher method becomes the matter of interest because in combination with the method of nonlinear normal vibration modes it allows to calculate steady forced vibrations in the system with multiple degrees of freedom (DOF) via reduction of the number of DOFs. However modern realizations of that approach have drawbacks such as iterative nature and the need to have initial approximation for the solution. The primary principle of Rauscher method is in obtaining periodic solutions of a non-autonomous system via studying some equivalent autonomous one.

In the paper a new non-iterative variant of Rauscher method is considered. In its current statement the method can be used in analysis of forced harmonic oscillations in a nonlinear system with one degree of freedom. The primary goals of the study were to find out what kind of equivalent autonomous systems could be built for a given non-autonomous one and how they can be used for the construction of periodic solutions and/or periodic phase plane orbits of the initial system.

It is shown that three different types of equivalent autonomous dynamical systems can be built for a given 1-DOF non-autonomous one. The system of $1^{\text {st }}$ type is a $4^{\text {th }}$ order dynamical system. Technically it can be considered as a 2-DOF system where additional "DOF" is explicitly "responsible" for forced oscillations. The system of $2^{\text {nd }}$ type is a $3^{\text {rd }}$ order dynamical system. Its periodic orbits are exactly the same as in the initial system. Using the invariant manifold of the system of $1^{\text {st }}$ type the system of $2^{\text {nd }}$ type can be reduced to the form $W\left(x, x^{\prime}\right)=0$ (which is called here the equivalent system of the $3^{\text {rd }}$ type).

It is important that the function $W\left(x, x^{\prime}\right)$ can be built a priori. Once $W\left(x, x^{\prime}\right)$ is found: (i) one can obtain different periodical orbits corresponding to forced oscillations in the initial system; (ii) one can estimate amplitudes of vibrations for these regimes; (iii) one can track bifurcations of periodical regimes of the initial system with respect to change of amplitude of external excitation $f$. As shown in the paper, periodical orbits of the initial non-autonomous system can be obtained via two different approaches: (i) as set of points on phase plane satisfying the condition $W\left(x, x^{\prime}\right)=0$;

(ii) via the application of harmonic balance method to the equivalent system of $1^{\text {st }}$ type using system's energy level as a continuation parameter. This approach has advantage over application of harmonic balance method to initial system because the latter requires good initial guess for expansion coefficients while the new approach does not and always starts from zero initial guess. 
Rauscher method, equivalent autonomous system, periodic solutions, invariant manifolds methodology, continuation techniques, harmonic balance method.

\section{Introduction}

The key principle of Rauscher method is that in certain modes non-autonomous dynamical system may behave like an autonomous one. So instead of studying a non-autonomous system one can study some equivalent autonomous system. Despite its concept was proposed in late 1930s [1] for 1-DOF nonlinear system and later extended [2], this method did not have much attention until recently, when it was combined with the concept of nonlinear normal vibration modes (NNMs) and therefore effectively extended for the case of multiple DOF system in the works by $\mathrm{Yu}$. Mikhlin, L. Manevich et al and K. Avramov [3-8] for studying steady near-resonance vibrations.

Modern realizations of Rauscher method incorporate reduction of the number of DOFs which is important for studying systems with large number of DOFs. But despite the present concepts of Rauscher method are already ready to studying multiple-DOF systems, they all have major drawbacks: (i) - iterative nature, (ii) initial approximation for solution for one of DOFs (which is not always known) is required (see Section 1). So making this method iteration-free would become a significant improvement.

In fact, when this approach is applied, at certain stage the initially nonautonomous dynamical system undergoing forced oscillations is substituted with some equivalent ("pseudoautonomous") one. This substitution is done in such way that some periodic solution(s) of the initial system satisfy the equations of the new autonomous one. So it appears that a non-autonomous dynamical system can be studied using some methods and approaches designed for studying autonomous dynamical systems (in the case above it is the NNM method used for reduction of the number of DOFs). This remarkable fact became another motivation for the present study.

The current paper is a proof-of-concept work which shows that periodic solutions corresponding to forced oscillations in a dissipative dynamical system can be 
obtained via studying an equivalent autonomous system without using iterative procedures (as opposed to existing approaches).

Please note that in the framework of this paper a non-autonomous (initial) and corresponding autonomous system are called equivalent if some (or all) periodic solutions of the initial system satisfy the equations of the equivalent autonomous one. The equivalent system, on the other hand, may be of different order and may have periodic solutions which do not satisfy the initial system. The primary points of interest in this study were the following:

i. What are the possible variants of equivalent autonomous systems that preserve forced periodical solutions of the initial system?

ii. Is it possible to construct equivalent system in such way that it does not introduce new periodic solutions (or at least periodical orbits on the phase plane) apart from existing ones?

iii. How can one construct periodic solutions of initial system and/or corresponding periodic orbits via studying an equivalent system without usage of iterative techniques?

In the framework of the current paper these questions were answered for the case of 1-DOF dissipative nonlinear system. Nevertheless, the author believes that the proposed approach, when used together with NNM concept can be extended to multiple-DOF case and may become a new method of studying forced oscillations in such systems. Thus, the present work can be considered a first step on this way. The 1-DOF mechanical system under consideration is the following:

$$
x^{\prime \prime}+h x^{\prime}+\omega^{2} x+\varphi(x)=f \cos (\Omega t)
$$

The system (1) is supposed to have single equilibrium position at $x=0$, the function $\varphi(x)$ is supposed to be an analytical function in the neighborhood of the equilibrium position and contain only nonlinear terms. Damping is considered to be small $\left(h<<\omega^{2}\right)$.

The paper is organized as follows. In the Section 1 current implementations of Rauscher method developed to date are considered and discussed. The concept of how one can create autonomous system from non-autonomous one is introduced. The rest of the paper represents the results obtained by the author and can be split into two main parts. In Section 2 of the paper different types of equivalent autonomous systems are considered and their properties are studied analytically. It 
is shown that one can develop three different types of equivalent systems. The system of $1^{\text {st }}$ type is a $4^{\text {th }}$ order dynamical system (technically it can be considered as a 2-DOF system where additional "DOF" is "responsible" for forced oscillations in (1)). The periodic orbits of this system correspond to all possible variations in amplitude of external excitation in the initial system. The system of $2^{\text {nd }}$ type is a $3^{\text {rd }}$ order dynamical system whose periodic orbits are exactly the same as in the initial system. Due to its complexity it is rather hard to build periodic orbits using the system of $2^{\text {nd }}$ type as it is. However, using invariant manifolds of the system of $1^{\text {st }}$ type the order of the system of $2^{\text {nd }}$ type can be reduced to 1 which creates equivalent system of $3^{\text {rd }}$ type. Its equation can be written as $W\left(x, x^{\prime}\right)=0$. The most remarkable is that this system provides only periodic orbits of the initial system and can be clearly represented geometrically: if one plots the surface $W\left(x, x^{\prime}\right)$, its zero levels become periodic orbits of the initial system. In fact, it is shown in Section 2 how to build the surface $W\left(x, x^{\prime}\right)$ analytically.

Section 3 contains alternative approach to building the whole surface $W\left(x, x^{\prime}\right)$ analytically. It is shown that the surface $W\left(x, x^{\prime}\right)$ can be built section-wise via application of harmonic balance method to the equivalent system of $1^{\text {st }}$ type using system energy level as a continuation parameter. This approach has advantage over application of harmonic balance method directly to initial system because the latter requires good initial guess for expansion coefficients while the new approach does not.

Since the paper is focused mostly on theoretical aspects, Section 4 contains two simple examples - Duffing equation with pure cubic and cubic-quadratic nonlinearity - working as proof-of-concept for the theoretical results presented here.

\section{Iterative variants of Rauscher method}

In this Section current implementations of Rauscher method developed to date are discussed. In its modern realization Rauscher method is developed for studying multiple-DOF systems and is, in fact, original Rauscher's concept of eliminating time from equations of motion combined with the method of nonlinear normal vibration modes (NNMs).

Let us consider, for example, non-autonomous dynamical system (1) which has $N$ degrees of freedom (DOFs): 


$$
\left\{x_{i}^{\prime \prime}+F_{i}\left(x_{1}, x_{2}, . ., x_{n}, x_{1}^{\prime}, x_{2}^{\prime}, . ., x_{n}^{\prime}, t\right)=0, \quad i=1 \ldots N\right.
$$

If the dependency $x_{k}=x_{k}(t)$ is somehow inverted to a form $t=t\left(x_{k}\right)$ ( $x_{k}$ is some pre-selected generalized coordinate), then the system (1) can be transformed into autonomous form:

$$
\left\{x_{i}^{\prime \prime}+F_{i}\left(x_{1}, x_{2}, . ., x_{n}, x_{1}^{\prime}, x_{2}^{\prime}, . ., x_{n}^{\prime}, t\left(x_{k}\right)\right)=0, \quad i=1 \ldots N\right.
$$

This idea of eliminating explicitly present time from equations of motion was presented by Rauscher in [1]. Originally the Rauscher method could be applied for 1-DOF systems only, namely, for finding steady forced oscillations. However, it was generalized for multi-DOF systems [2-8] by means of theory of nonlinear normal vibration modes (NNMs).

The dependency $t=t\left(x_{k}\right)$ is constructed in such way that the systems (2) and (3) have the same solutions corresponding to steady forced oscillations (in this sense (2) can be called an equivalent system to (1)). For conservative systems (or close to conservative ones) the dependency $t=t\left(x_{k}\right)$ can be represented in the form of quadratures (see for example $[3,4]$ ).

In the papers $[6,7]$ another approach for elimination of time $t$ is proposed. Equations of motion are supposed to be:

$$
\left\{x_{i}^{\prime \prime}+F_{i}\left(x_{1}, x_{2}, \ldots, x_{n}\right)=f_{i} \cos (\Omega t),(i=1 \ldots N)\right. \text {. }
$$

Then time $t$ can be eliminated, according to [6,7], by means of constructing a dependency

$$
\cos (\Omega t)=a_{0}+a_{1} x_{k}+a_{2} x_{k}^{2}+a_{3} x_{k}^{3}+\ldots
$$

or $\cos (\Omega t)=C\left(x_{k}\right)$. Such expansion can be constructed if some initial approximation for $x_{k}$ is known in form $x_{k}=A_{0}+A_{1} \cos (\Omega t)+A_{2} \cos (2 \Omega t)+\ldots$ It is proposed in [6] to rewrite the latter using trigonometric transformations as $x_{k}=A_{0}-A_{2}+\left(A_{1}-3 A_{3}\right) \cos (\Omega t)+2 A_{2} \cos ^{2}(\Omega t)+4 A_{3} \cos ^{3}(\Omega t) \ldots$ Once (5) is substituted into this expression, coefficients of the same orders of $x_{k}$ are equated, which leads to a system of algebraic equations that can be solved for unknowns $a_{0}, a_{1}, a_{2},$. 
Once current approximation for $a_{0}, a_{1}, a_{2}, .$. is known, expression (5) is substituted into (4). The new system does not contain time $t$ explicitly:

$$
\left\{x_{i}^{\prime \prime}+F_{i}\left(x_{1}, x_{2}, . ., x_{n}\right)=f_{i}\left(a_{0}+a_{1} x_{k}+a_{2} x_{k}^{2}+a_{3} x_{k}^{3}+\ldots\right),(i=1 \ldots N)\right.
$$

It is analyzed via invariant manifolds methodology. If state space variables of a nonlinear system undergo coherent changes, one can speak of what is called nonlinear normal mode (NNM). NNM in the system (6) can be expressed as a set of such dependencies:

$$
\left\{\begin{array}{l}
x_{i}=x_{i}\left(x_{k}, x_{k}^{\prime}\right) \\
x_{i}^{\prime}=x_{i}^{\prime}\left(x_{k}, x_{k}^{\prime}\right)
\end{array}, i=1, . . k-1, k+1, . . N .\right.
$$

These dependencies are invariant manifolds of the autonomous system (6) (they are also called NNMs by Shaw and Pierre). Geometrically they represent a set of surfaces (or a single hyper-surface) in the state space. In order to find NNM the autonomous system should be transformed into a system of partial differential equations taking $x_{k}$ and $x_{k}^{\prime}$ as couple of independent variables. This approach was developed by S. Shaw and C. Pierre in $[9,10]$. Comprehensive overview of different NNM theories can be found in $[11,12]$.

When (7) is constructed from (6), the system (4) can be reduced to 1-DOF with respect to $x_{k}$. This allows one to obtain more precise trigonometrical approximation for $x_{k}$ and therefore iterative process is constructed. This approach was extended by the author for more general case:

$\left\{x_{i}^{\prime \prime}+F_{i}\left(x_{1}, x_{2}, . ., x_{n}, x_{1}^{\prime}, x_{2}^{\prime}, . ., x^{\prime}, t\right)=f_{i} \cos (\Omega t)+g_{i} \sin (\Omega t), \quad i=1 \ldots N\right.$

In order to make the system (8) non-autonomous one needs here to build such dependencies:

$$
\begin{aligned}
& \cos (\Omega t)=C\left(x_{k}, x_{k}^{\prime}\right) \\
& \sin (\Omega t)=S\left(x_{k}, x_{k}^{\prime}\right)
\end{aligned}
$$

In the papers [13-16] the latter are represented in the form of power series. It also should be noted that different forms and various applications of the Rauscher method are discussed in $[11,17]$. Among recent works where the Rauscher method is used one can find the following ones: [18, 19].

Thus, appealing feature of Rauscher method in its modern variants is reduction of number of DOFs due to usage of NNMs. But as it clear from the above the 
methods described in this Section have a major drawback - iterative nature. So the goal of the present paper is to find a non-iterative approach which corresponds to the primary idea of the Rauscher method: eliminating time from non-autonomous equations of motion in order to construct an equivalent autonomous system of equations which has the same periodic solutions as the initial one.

The present paper addresses this issue only for the case of 1-DOF dynamical system so far. Further developments will be required.

\section{Equivalent Autonomous Systems and Their Properties}

\subsection{A linear system}

As a starting point for all considerations the linearized system (1) is discussed here. If the system (1) is linear $(\varphi(x)=0)$, then finding Rauscher approximations is quite an easy task.

In such case one has: $x^{\prime \prime}+h x^{\prime}+\omega^{2} x=f \cos (\Omega t)$ and $x^{\prime \prime \prime}+h x^{\prime \prime}+\omega^{2} x^{\prime}=-f \Omega \sin (\Omega t)$. If only periodic solutions are considered then the following relations take place: $x^{\prime \prime}=-\Omega^{2} x, x^{\prime \prime \prime}=-\Omega^{2} x^{\prime}$ since $x=A \cos (\Omega t)+B \sin (\Omega t)$ for such case. Thus $x^{\prime \prime}$ and $x^{\prime \prime \prime}$ can be eliminated which yields the following Rauscher approximations:

$$
\cos (\Omega t)=\frac{1}{f}\left(h x^{\prime}+\left(\omega^{2}-\Omega^{2}\right) x\right) ; \quad \sin (\Omega t)=-\frac{1}{\Omega f}\left(\left(\omega^{2}-\Omega^{2}\right) x^{\prime}-h \Omega^{2} x\right)
$$

It should also be noted that expression $x^{\prime \prime}+\Omega^{2} x=0$ is true for every value of amplitude or phase of external load if one considers only periodic solutions. So this expression can be considered as some kind of equivalent autonomous system which shares solutions with initial non-autonomous one. Though this equation does not allow one to find particular periodic solution $x(t)$, Rauscher approximations (10) allow that.

Periodical solution $x(t)$ can be found only by means of the Rauscher expansions when they are solved for $x$ and $x^{\prime}$ as a system of linear algebraic equations. Moreover, if one substitutes (10) into trigonometric identity 
$\cos ^{2}(\Omega t)+\sin ^{2}(\Omega t)=1$ it results in equation of periodical orbit on the phase plane which corresponds to forced oscillations:

$$
\left(h^{2} \Omega^{2}+\left(\omega^{2}-\Omega^{2}\right)^{2}\right)\left(x^{2}+\frac{\left(x^{\prime}\right)^{2}}{\Omega^{2}}\right)-f^{2}=0
$$

or

$$
W\left(x, x^{\prime}\right)=0
$$

One can note that:

1. Higher derivatives of displacement can be evaluated via displacement and corresponding velocity in the regime of forced oscillations;

2. The latter can be used for the reduction of the system's order and for the construction of Rauscher expansions in the form (9);

3. It is possible to transform dynamical system to the form $W\left(x, x^{\prime}\right)=0$ and in fact, it appears that trajectory corresponding to forced oscillations is the line of intersection of surface $W$ and coordinate plane (Fig. 1);

4. The $W$ surface can be built a priori without calculation of any solution of non-autonomous system.

In the subsequent sections these remarkable statements are extended to nonlinear systems of type (1).

\subsection{Equivalent dynamical system of $1^{\text {st }}$ type}

If the system $(1)$ is nonlinear $(\varphi(x) \neq 0)$, the relations $x^{\prime \prime}=-\Omega^{2} x, x^{\prime \prime \prime}=-\Omega^{2} x^{\prime}$ require some correcting function $y(t)$ to be introduced:

$x^{\prime \prime}=-\Omega^{2} x+y, x^{\prime \prime \prime}=-\Omega^{2} x^{\prime}+y^{\prime}$.

Therefore an additional equation for $y(t)$ is required. In order to find it let us

differentiate (1) twice with respect to time $t$ and then add term-wise equation (1) multiplied by $\Omega^{2}$ to the result:

$$
x^{I V}+\Omega^{2} x^{\prime \prime}+h\left(x^{\prime \prime \prime}+\Omega^{2} x^{\prime}\right)+\omega^{2}\left(x^{\prime \prime}+\Omega^{2} x\right)+\Omega^{2} \varphi+\varphi_{x x}^{\prime \prime} \cdot\left(x^{\prime}\right)^{2}+\varphi_{x}^{\prime} x^{\prime \prime}=0
$$

After change of variables this equation can be written in another way: 


$$
\left\{\begin{array}{l}
x^{\prime \prime}+\Omega^{2} x=y \\
y^{\prime \prime}+h y^{\prime}+\omega^{2} y+\Phi\left(x, x^{\prime}, y\right)=0
\end{array}\right.
$$

where $\Phi\left(x, x^{\prime}, y\right)=\Omega^{2} \varphi+\varphi_{x x}^{\prime \prime} \cdot\left(x^{\prime}\right)^{2}+\varphi_{x}^{\prime} \cdot\left(y-\Omega^{2} x\right)$.

It should be noted that (13) and (14) are essentially the same and share exactly the same solutions. Both (13) and (14) represent a dynamical system which is equivalent to the initial system (1). Technically the system (14) can be considered as a 2-DOF system where the additional "virtual" degree of freedom corresponds to oscillations occurring with driving frequency $\Omega$.

Since this new system has higher order than the initial one, it has more solutions than the initial one. Let us analyze these solutions.

Initial equation (1) can be represented in the following form:

$$
F\left(x^{\prime \prime}, x^{\prime}, x\right)=f \cos (\Omega t)
$$

Therefore (13) can be written as $F^{\prime \prime}+\Omega^{2} F=0$. This equation can be easily solved for $F: F\left(x^{\prime \prime}, x^{\prime}, x\right)=a \cos (\Omega t+\psi)$ where $a$ and $\psi$ are arbitrary constants. Thus it can be concluded from this solution that all possible solutions of equivalent systems (13) and (14) correspond to various possible combinations of amplitude and phase of external excitation. Among others the solutions of autonomous system $F\left(x^{\prime \prime}, x^{\prime}, x\right)=0$ are also included in that set (case $\left.a=0\right)$.

It can be noted that changes in $\psi$ do not affect shape of periodical orbits of the solutions of the equation $F\left(x^{\prime \prime}, x^{\prime}, x\right)=a \cos (\Omega t+\psi)$. Thus, even more important conclusion arises:

$\Delta$ Statement 1. All possible periodic orbits on the phase plane $\left(x, x^{\prime}\right)$ and in the state space $\left(x, x^{\prime}, y, y^{\prime}\right)$ found by means of equivalent systems (13) or (14) correspond to various values of a single parameter - amplitude $f$ of external excitation.

For convenience equivalent systems (13) or (14) will be called equivalent systems of $1^{\text {st }}$ type in the subsequent considerations. 


\subsection{Equivalent dynamical system of $2^{\text {nd }}$ type}

Differentiating (15) with respect to time $t$ yields: $\left[F\left(x^{\prime \prime}, x^{\prime}, x\right)\right]^{\prime}=-\Omega f \sin (\Omega t)$. Taking both this equation and (15) one can obtain: $\left(F^{\prime}\right)^{2}+\Omega^{2} F^{2}=\Omega^{2} f^{2}$ (via relation $\left.\cos ^{2}(\Omega t)+\sin ^{2}(\Omega t)=1\right)$ or in extended form :

$$
\left(x^{\prime \prime \prime}+h x^{\prime \prime}+\omega^{2} x^{\prime}+\varphi_{x}^{\prime} \cdot x^{\prime}\right)^{2}+\Omega^{2}\left(x^{\prime \prime}+h x^{\prime}+\omega^{2} x+\varphi(x)\right)^{2}=\Omega^{2} f^{2}
$$

Equation $\left(F^{\prime}\right)^{2}+\Omega^{2} F^{2}=\Omega^{2} f^{2}$ can be solved for $F$ as $F\left(x^{\prime \prime}, x^{\prime}, x\right)=f \cos (\Omega t+\psi)$ where $\psi$ is an arbitrary constant. It can be concluded from such solution that all possible solutions of the equivalent system (16) correspond to all possible variations of the phase of external excitation. Thus this new system has the same periodic orbits as the initial one.

\section{$\Delta$ Statement 2. All possible periodic orbits on the phase plane $\left(x, x^{\prime}\right)$ found} by means of equivalent system (16) are exactly the same as in the initial system (1).

For convenience the equivalent system (16) will be called the equivalent system of $2^{\text {nd }}$ type in the subsequent considerations.

Since the equation of the equivalent system of $2^{\text {nd }}$ type is of higher order than (1) and has complex structure, it is rather hard to use it as it is for construction of periodic orbits of initial system. However, this type of equivalent systems does not introduce additional periodic orbits to those existing in initial system. So this important fact must be used.

Since the study is focused only on finding periodic regimes, it can be noticed that in such case expressions $x, x^{\prime}, x^{\prime \prime}, x^{\prime \prime \prime}$ change in time in coherent manner.

Therefore, they are interconnected and can be expressed via one another. This will allow to eliminate $x^{\prime \prime}, x^{\prime \prime \prime}$ from (16) while preserving its important property declared in Statement 2. Functional dependency between $x^{\prime \prime}, x^{\prime \prime \prime}$ and $x, x^{\prime}$ can be established by means of the analysis of the equivalent system of $1^{\text {st }}$ type (14). Indeed, since $x^{\prime \prime}=-\Omega^{2} x+y, x^{\prime \prime \prime}=-\Omega^{2} x^{\prime}+y^{\prime}$, then one needs to uncover dependency of $y$ and $y^{\prime}$ on $x, x^{\prime}$ in forced oscillations mode. This can be done via application of invariant manifolds methodology to the equivalent system of $1^{\text {st }}$ type because technically it is a 2-DOF system. 


\subsection{Equivalent system investigation via invariant manifolds methodology}

When investigation of system (1) is focused on periodical solutions only, it can be noted that the solution itself and all its derivatives change in time in a coherent manner. Therefore one may expect to find the following dependencies in (14):

$$
\left\{\begin{array}{c}
y=y\left(x, x^{\prime}\right) \\
y^{\prime}=z=z\left(x, x^{\prime}\right)
\end{array}\right.
$$

Finding these would allow one to exclude auxiliary function $y(t)$. Following [10] these dependencies can be constructed using invariant manifolds methodology. It can be applied to the equivalent system of 1 st type because it consists of two ODEs of $2^{\text {nd }}$ order.

Let us denote $x=u, x^{\prime}=v$. The system (14) is now written in standard form:

$$
\left\{\begin{array}{l}
u^{\prime}=v \\
v^{\prime}=-\Omega^{2} u+y \\
y^{\prime}=z \\
z^{\prime}=-\left(h y^{\prime}+\omega^{2} y+\Phi(u, v, y)\right)
\end{array}\right.
$$

Differentiation with respect to time $t$ now becomes a partial differential operator: $\frac{d}{d t}=v \frac{\partial}{\partial u}+v^{\prime} \frac{\partial}{\partial v}$, which leads to the following PDEs:

$$
\left\{\begin{array}{l}
u \frac{\partial y}{\partial u}+\left(y-\Omega^{2} u\right) \frac{\partial y}{\partial v}=z \\
u \frac{\partial z}{\partial u}+\left(y-\Omega^{2} u\right) \frac{\partial z}{\partial v}=-\left(h z+\omega^{2} y+\Phi(u, v, y)\right)
\end{array}\right.
$$

Such PDEs can be solved in different ways (the solution can be written in form of power series [10] or found via Galerkin method [20]). Here the solution is found in power series form:

$$
\left\{\begin{array}{l}
y=a_{1} u+a_{2} v+a_{3} u^{2}+a_{4} u v+a_{5} v^{2}+\ldots \\
z=b_{1} u+b_{2} v+b_{3} u^{2}+b_{4} u v+b_{5} v^{2}+\ldots
\end{array}\right.
$$

Solution (20) is substituted into (19). (At this stage the functions $\Phi(u, v, y)$ and $\varphi(x)$ are considered to be polynomials or they should be expanded in power 
series otherwise). When terms of the same power of $u$ and $v$ are equated in the obtained equalities this leads to a recurrent system of algebraic equations with respect to unknown coefficients $a_{i}, b_{i}$. Among others there exists a closed subsystem of nonlinear equations with respect to $a_{1}, a_{2}, b_{1}, b_{2}$.

All other equations in the recurrent system are linear with respect to unknowns of current step but nonlinear with respect to quantities, evaluated previously. That is, there can be found system of linear algebraic equations with respect to $a_{3}, a_{4}, a_{5}, b_{3}, b_{4}, b_{5}$ (coefficients of quadratic terms). Its matrix is constant but right hand side depends on previously evaluated $a_{1}, a_{2}, b_{1}, b_{2}$. The same for coefficients of cubic terms and so on. This means that once $a_{1}, a_{2}, b_{1}, b_{2}$ are found, all other coefficients are evaluated in a unique way.

Equations with respect to $a_{1}, a_{2}, b_{1}, b_{2}$ are:

$$
\left\{\begin{array}{l}
a_{1} a_{2}-\Omega^{2} a_{2}=b_{1} \\
b_{2} a_{1}-\Omega^{2} b_{2}=-h b_{1}-\omega^{2} a_{1} \\
a_{1}+a_{2}^{2}=b_{2} \\
b_{1}+b_{2} a_{2}=-h b_{2}-\omega^{2} a_{2}
\end{array}\right.
$$

Consecutive elimination of unknowns produces an equation of the 6-th degree with respect to $a_{1}$ :

$$
\begin{aligned}
& a_{1}\left(a_{1}-\Omega^{2}+\omega^{2}\right)\left[a_{1}^{4}-4 \Omega^{2} a_{1}^{3}+\Omega^{2}\left(6 \Omega^{2}-2 \omega^{2}+h^{2}\right) a_{1}^{2}+\right. \\
& \left.+2 \Omega^{4}\left(2\left(\omega^{2}-\Omega^{2}\right)-h^{2}\right) a_{1}+\Omega^{4}\left(\Omega^{2} h^{2}+\left(\Omega^{2}-\omega^{2}\right)^{2}\right)\right]=0
\end{aligned}
$$

When solved, this equation allows one to find two real roots (in the small damping case $\left.h \ll \omega^{2}\right)$ :

- $a_{1}=\Omega^{2}-\omega^{2}$ This is a parasitic solution. After all necessary transformations and back-substitutions it produces the expansion $y=-h x^{\prime}-\omega^{2} x+\Omega^{2} x-\varphi(x)$. Taking into account that $y=x^{\prime \prime}+\Omega^{2} x$ this leads to equation $x^{\prime \prime}=-h x^{\prime}-\omega^{2} x-\varphi(x)$ which is the initial system (1) with zero external load. So this solution corresponds to free oscillations, not forced ones.

- $a_{1}=0$ - when substituted into other equations this root leads to the trivial solution of the system (21): $\left\{a_{1}=a_{2}=b_{1}=b_{2}=0\right\}$ - when substituted further, but this solution allows one to find nonzero $a_{3}, a_{4}, a_{5}, b_{3}, b_{4}, b_{5}$ and so on. 
The above analysis leads to an important conclusion:

$\Delta$ Statement 3. In the state space $\left(x, x^{\prime}, y, y^{\prime}\right)$ there exist unique hypersurface (17) which corresponds to forced oscillations in the initial system (1) and passes through the equilibrium position of that system in the space of variables $\left(x, x^{\prime}, y, y^{\prime}\right)$.

Consider now periodic solutions of the initial system (1) and their connection with the above-mentioned hypersurface. If amplitude of external excitation $f$ is varied, periodic orbits form some hypersurface in the space of variables $\left(x, x^{\prime}, y, y^{\prime}\right)$. This surface passes through the equilibrium position (see Figure 2). Taking into account that periodic solutions of (1) are at the same time the solutions of the equivalent system (14) one can conclude that hypersurface formed by periodic orbits is exactly the same hypersurface that is mentioned in Statement 3. That is:

$\Delta$ Statement 4. For each point on the surface (17) which satisfy equations (19) there exist a closed trajectory in the space of variables $\left(x, x^{\prime}, y, y^{\prime}\right)$ which passes through that point and corresponds to a periodic solution of (1) under some value of external excitation $f$.

It follows from the above that the dependencies (17) can be built by means of finding periodical solutions for different values of $f$ and interpolation through the obtained results (Figure 2). However, there exist more convenient approach which is described in Section 3.

\subsection{Rauscher expansions construction. Equivalent dynamical system of $3^{\text {rd }}$ type.}

It can be derived from (1) that $\cos (\Omega t)=\left(x^{\prime \prime}+h x^{\prime}+\omega^{2} x+\varphi\right) / f$ and $\sin (\Omega t)=-\left(x^{\prime \prime \prime}+h x^{\prime \prime}+\omega^{2} x^{\prime}+\varphi_{x}^{\prime} \cdot x^{\prime}\right) / \Omega f$. Taking into account dependencies (17) obtained via the equivalent system of $1^{\text {st }}$ type one has the following Rauscher approximations:

$$
\left\{\begin{array}{l}
\cos (\Omega t)=\frac{1}{f}\left(y\left(x, x^{\prime}\right)+h x^{\prime}+\left(\omega^{2}-\Omega^{2}\right) x+\varphi\right) \\
\sin (\Omega t)=-\frac{1}{\Omega f}\left(z\left(x, x^{\prime}\right)+h\left(y\left(x, x^{\prime}\right)-\Omega^{2} x\right)+\left(\omega^{2}-\Omega^{2}\right) x^{\prime}+\varphi_{x}^{\prime} \cdot x^{\prime}\right)
\end{array}\right.
$$

which exactly corresponds to the form (8) used by other Rauscher-like methods. 
In fact, the system (22) can be considered as a couple of algebraic equations with respect to $x$ and $x^{\prime}$.The solution of this couple of equations - $x$ and $x^{\prime}-$ depends on $t$ as it is a parameter which is introduced in (22) through periodical functions. This leads to a remarkable conclusion that $x$ and $x^{\prime}$ can only be periodical functions if the dependencies (22) take place simultaneously.

Taking into account the identity $\cos ^{2}(\Omega t)+\sin ^{2}(\Omega t)=1$ one can obtain from (22):

$$
\begin{aligned}
& \frac{1}{\Omega^{2}}\left(z\left(x, x^{\prime}\right)+h\left(y\left(x, x^{\prime}\right)-\Omega^{2} x\right)+\left(\omega^{2}-\Omega^{2}\right) x^{\prime}+\varphi_{x}^{\prime} \cdot x^{\prime}\right)^{2}+ \\
& +\left(y\left(x, x^{\prime}\right)+h x^{\prime}+\left(\omega^{2}-\Omega^{2}\right) x+\varphi\right)^{2}=f^{2}
\end{aligned}
$$

This expression is not changed if the phase of external load is varied by an arbitrary constant.

This equivalent system of a new type can be also considered as the result of substitution of (17) into the system of $2^{\text {nd }}$ type (16) and division of the latter by $\Omega^{2}$. Thus, the Statement 2 is correct for this new system (23) as well: all possible periodic orbits on the phase plane found by means of equivalent system (23) are exactly the same as in the initial system (1). Moreover, due to the considerations above all possible solutions of the equivalent system (23) are exclusively periodic solutions of the initial system (1) which correspond to various values of the phase of external load.

$\Delta$ Statement 5. The only trajectories on the phase plane $\left(x, x^{\prime}\right)$ which can be found by means of the equivalent system (23) are periodic orbits of the initial system (1).

The equivalent system (23) will be called the equivalent system of 3rd type. In the next Section the system (23) receives clear geometrical interpretation.

\subsection{Geometrical interpretation of the equivalent system of $3^{\text {rd }}$ type.}

Let us denote left hand side of (23) as $W_{0}$ and left-hand side minus right-hand side of (23) as $W$ :

$$
\begin{gathered}
W_{0}=\frac{1}{\Omega^{2}}\left(z\left(x, x^{\prime}\right)+h\left(y\left(x, x^{\prime}\right)-\Omega^{2} x\right)+\left(\omega^{2}-\Omega^{2}\right) x^{\prime}+\varphi_{x}^{\prime} \cdot x^{\prime}\right)^{2}+ \\
+\left(y\left(x, x^{\prime}\right)+h x^{\prime}+\left(\omega^{2}-\Omega^{2}\right) x+\varphi\right)^{2} \\
W=W_{0}-f^{2}
\end{gathered}
$$


In such case it is clear that equation (23) is true only when $W=0$. Therefore periodic orbits of the system (1) are the lines of intersection of function $\mathrm{W}$ and coordinate plane (Fig. 3,a).

On the other hand, it is clear from (24) that equation (23) is true when $\sqrt{W_{0}}=|f|$. Therefore level curves of the surface $\sqrt{W_{0}}$ are periodic orbits of the system (1) corresponding to different levels of external excitation (Fig. 3,b). Similar statement is correct for $W$ too.

The conditions $W=0$ and $\sqrt{W_{0}}=|f|$ can be treated as a certain type of periodicity conditions for the solutions of the initial system. It also should be noted here that surfaces $W$ and $W_{0}$ possess certain invariance properties with respect to forced periodic motions in (1). In particular, while all parameters of the system are fixed, different periodic motions of the system correspond to the same set of level curves of these surfaces (of the same level).

It is clear that once $W$ and $W_{0}$ are carefully computed:

- one can obtain different periodical orbits which correspond to forced oscillations in (1);

- one can estimate amplitudes of vibrations for these regimes;

- one can track bifurcations of periodical solutions of (1) with respect to change of amplitude of external excitation $f$.

- when represented graphically, the condition $\sqrt{W_{0}\left(x, x^{\prime}\right)}=|f|$ shows exact correspondence between the amount of the external excitation $f$ and the number and shape of existing periodical orbits of the system.

Thus the functions $W$ and $W_{0}$ may be very useful visual aids that help to understand behavior of the dynamical system under investigation. These functions can be constructed a priori via invariant manifold of the equivalent system of $1^{\text {st }}$ type.

\section{Investigation via harmonic balance method. Construction of $\mathbf{W}$ and $W_{0}$ via level curves.}

The current Section provides alternative view on how the surfaces $W$ and $W_{0}$ can be constructed and how periodic solutions of initial system can be obtained using equivalent autonomous systems. 
If the surfaces (17) are obtained analytically one can use expressions (24) to fully specify $W$ and $W_{0}$. However, if some periodic solution $x(t)$ in (14) is found then one can build a single corresponding section (level curve) of $W$ and $W_{0}$ since $W\left(x, x^{\prime}\right)=$ const,$W_{0}\left(x, x^{\prime}\right)=$ const for every such solution (Fig. 4). So $W$ and $W_{0}$ can be obtained section by section via their level curves. One can then interpolate through the obtained sections. This is an alternative approach to solving the problem of $W$ and $W_{0}$ construction.

Such approach can be useful if one desires to study large-amplitude motions. In this case evaluation of (17) via power series may not be accurate enough while other methods of solving PDEs (19) may require some sophisticated computations.

Since the level curves of $W$ and $W_{0}$ are closed curves corresponding to various periodic orbits of the equivalent dynamical system of $1^{\text {st }}$ type (14), harmonic balance method can be engaged to calculation of such orbits in many cases. It will be shown further that application of harmonic balance method to the equivalent system of $1^{\text {st }}$ type (14) has advantage over application of this method directly to initial system because the latter requires good initial guess for expansion coefficients while the new approach does not. When multiple solutions are to be found the usage of equivalent system also provides benefits (see Remark 1 in the next Subsection).

\subsection{Application of harmonic balance method to equivalent dynamical system of $1^{\text {st }}$ type}

The solution of the equivalent dynamical system of $1^{\text {st }}$ type (14) can be written as a truncated Fourier series taking into account harmonics up to n-th order:

$$
\begin{aligned}
& x \approx A_{0}+A_{1} \cos (\Omega t)+\sum_{k=2}^{n} A_{k} \cos (k \Omega t)+B_{k} \sin (k \Omega t) \\
& y \approx \Omega^{2}\left(A_{0}+\sum_{k=2}^{n}\left(1-k^{2}\right)\left(A_{k} \cos (k \Omega t)+B_{k} \sin (k \Omega t)\right)\right)
\end{aligned}
$$

Note that due to arbitrariness in choosing time reference point for (14) the expression (25) is chosen is such way that it does not contain term $B_{1} \sin (\Omega t)$. The first equation of (14) is satisfied by (25) automatically. If relations (25) are substituted into the second equation of (14), one can obtain $2 n-1$ algebraic 
equations of harmonic balance method with respect to unknowns $A_{k}, B_{k}$. The number of equations is $2 n-1$, not $2 n$ as it may be expected.

An additional equation is required here in order to select some particular periodic trajectory in the state space $\left(x, x^{\prime}, y, y^{\prime}\right)$ among others. It is proposed here to construct this additional equations in the following way: $\bar{h}\left(x, x^{\prime}\right)=\lambda$ taking into account (25). Supposing that value of $\lambda$ differs from trajectory to trajectory one can apply some continuation techniques for obtaining sections of $W$ surface. It was discussed above that varying amplitude of external excitation $f$ allows construction of $W$ surface section-wise. However, making $f$ a continuation parameter is not convenient. Consider the following example.

Three sample amplitude-frequency responses which correspond to different values of $f$ are presented on Fig. 5a. If one fixates frequency value at $\Omega=\Omega_{0}$ seven periodical regimes can be found here. Plotting the corresponding trajectories on the $\left(x, x^{\prime}\right)$ plane (Fig. 5b) shows that transfer from innermost to outermost trajectory requires one to change $f$ in non-monotonic way: increase, decrease, increase again. This causes inconvenience if some continuation techniques with respect to $f$ are used.

Therefore in the present work it is proposed to build expression $\bar{h}\left(x, x^{\prime}\right)=\lambda$ considering $\bar{h}$ as averaged total energy of the system per period which can be written in the following form:

$$
\bar{h}(t)=\frac{\Omega}{2 \pi} \int_{t}^{t+\frac{2 \pi}{\Omega}}\left[T\left(x^{\prime}(\tau)\right)+\Pi(x(\tau))\right] d \tau
$$

where $T$ and $\Pi$ - are kinetic and potential energy respectively. If periodic motion $x(t)$ with period $\frac{2 \pi}{\Omega}$ is considered then $\bar{h}(t)=$ const $\forall t$. So the particular value $\lambda$ of averaged total energy per period $\bar{h}$ can be taken as a continuation parameter for evaluation of periodic trajectories.

Complete system of algebraic equations of harmonic balance method can be written as follows:

$$
\left\{\begin{array}{l}
\left.\Phi_{i}\left(A_{0} \ldots A_{n}, B_{2} \ldots B_{n}\right)=0\right\},(i=1 \ldots 2 n-1) \\
\bar{h}\left(x, x^{\prime}\right)=\lambda
\end{array}\right.
$$


Equations (27) can be written in general form as $\left\{\Phi_{i}([A], \lambda)=0,(i=1 \ldots 2 n)\right.$ where $[A]=\left\{A_{0} \ldots A_{n}, B_{2} \ldots B_{n}\right\}^{T}=\left\{a_{1}, \ldots, a_{2 n}\right\}^{T}$ - vector of unknowns.

Now some continuation technique can be applied to (27) in order to find the solution $[A]$. Or equations (27) may be transformed into differential form with respect to independent variable $\lambda$ and solved via numerical integration.

Once $A_{0} \ldots A_{n}, B_{2} \ldots B_{n}$ are obtained for some fixated value of $\lambda$, one can evaluate $x(t)$ and then build corresponding section of the $W$ and $W_{0}$ surfaces.

Remark 1. Please, note that continuation via $\lambda$ applied to equivalent system of $1^{\text {st }}$ type has advantage over direct application of harmonic balance method. Consider amplitude-frequency response, depicted on Fig. 6a,b. At some particular value of excitation frequency $\Omega^{*}$ there exist three periodic regimes. In order to obtain them via just harmonic balance method one may need to use continuation along the whole frequency response curve (Fig. 6a) since initial approximations for amplitudes of these three regimes are unknown. Due to bifurcation points and turning points that type of continuation may not be easy. On the other hand, if one applies continuation with respect to $\lambda$ as described above, the starting value for continuation parameter and unknown amplitudes are always known - zeroes. Gradually increasing $\lambda$ from zero one can find all three periodic regimes subsequently (Fig. 6b).

It also should be noted that during continuation via $\lambda$ all the intermediate solutions found using this method belong to the invariant manifold of equivalent system (Fig. 6d). This is not correct for application of harmonic balance method to the initial system (Fig. 6c).

Remark 2. Since $x(t)$ obtained via (25-27) is an approximate periodic solution of (14), the conditions $W\left(x, x^{\prime}\right)=$ const,$W_{0}\left(x, x^{\prime}\right)=$ const are not fully satisfied. The result of substitution of an approximate solution $x(t)$ into (24) produces constant plus some small oscillating function (residual). Therefore instead of real level curves one obtains a set of "wavy" closed curves (see for example Fig. 14,a). If large enough number of harmonics $n$ is taken, then residual becomes sufficiently small and the obtained results can be simply averaged over oscillations' period for each section of the surface (Fig. 14,b).

Let us denote averaged values of $W$ and $W_{0}$ as $\bar{w}$ and $\bar{w}_{0}$ respectively. 


\subsection{Calculation of solutions of the initial system}

Each section on the $W$ surface has corresponding periodic solution $x(t)$ of the system (14). In turn, each such solution has corresponding value of continuation parameter $\lambda$. On the other hand each section on the $W$ surface has corresponding constant value $\bar{w}$. Therefore there exists dependency between $\bar{w}$ and $\lambda$ : $\bar{w}=\bar{w}(\lambda)$. This dependency has the same major property as function $W$ : if the periodic orbit corresponding to $x(t)$ is at the same time a periodic orbit of the initial system $(1)$, then $\bar{w}(\lambda)=0$.

This means that there is no need in actual construction and plotting of the $W$ surface. Once continuation process with respect to $\lambda$ is applied to (27), it is nesessary to calculate corresponding value of $\bar{w}$ for each new value of $\lambda$. When zero value of $\bar{w}$ is found, it means that corresponding solution $x_{\text {appr }}(t)$ of equivalent system forms on the phase plane a periodic orbit which is at the same time periodic orbit of initial system.

However, $x_{\text {appr }}(t)$ is a solution of equivalent system (14) but it is not an actual solution of the initial system (1). They are represented by the same periodic orbit in phase space but have different time reference points. This happens due to arbitrariness in choosing time reference point for (14) because that system is an autonomous one.

So actual solution of (1) is $x_{a}(t)=x_{\text {appr }}\left(t-t_{\text {corr }}\right)$ where $t_{\text {corr }}$ is some correcting timeshift. $x_{a}(t)$ satisfies initial equation (1) (written here in form (15)): $F\left(x_{a}^{\prime \prime}, x_{a}^{\prime}, x_{a}\right)=f \cos (\Omega t)$.

It follows from the above that:

$$
F\left(x_{\text {appr }}^{\prime \prime}(t), x_{\text {appr }}^{\prime}(t), x_{\text {appr }}(t)\right)=f \cos \left(\Omega t+\psi_{\text {corr }}\right)
$$

where $\psi_{\text {corr }}=\Omega t_{\text {corr }}$. Let us introduce mean square residual of the equation (28):

$$
\delta\left(\psi_{\text {corr }}\right)=\frac{\Omega}{2 \pi} \int_{0}^{2 \pi / \Omega}\left(F\left(x_{\text {appr }}^{\prime \prime}(t), x_{\text {appr }}^{\prime}(t), x_{\text {appr }}(t)\right)-f \cos \left(\Omega t+\psi_{\text {corr }}\right)\right)^{2} d t
$$

so $\psi_{\text {corr }}=\arg \min _{\psi \in(-\pi, \pi]} \delta$. Necessary minimum condition of (29) $d \delta / d \psi_{\text {corr }}=0$ yields: 


$$
\tan \left(\psi_{\text {corr }}\right)=-\frac{\int_{0}^{2 \pi / \Omega} F\left(x_{\text {appr }}^{\prime \prime}(t), x_{\text {appr }}^{\prime}(t), x_{\text {appr }}(t)\right) \sin (\Omega t) d t}{\int_{0}^{2 \pi / \Omega} F\left(x_{\text {appr }}^{\prime \prime}(t), x_{\text {appr }}^{\prime}(t), x_{\text {appr }}(t)\right) \cos (\Omega t) d t}
$$

Thus the actual solution can be fully reconstructed.

\section{Examples}

As an example Duffing equation is considered:

$m x^{\prime \prime}+\beta x^{\prime}+c x+\tilde{\gamma}_{1} x^{2}+\tilde{\gamma}_{2} x^{3}=\tilde{f} \cos (\Omega t)$.

It can be written in the form (1):

$$
x^{\prime \prime}+h x^{\prime}+\omega^{2} x+\gamma_{1} x^{2}+\gamma_{2} x^{3}=f \cos (\Omega t)
$$

where $h=\beta / m, \omega^{2}=c / m, \gamma_{1}=\tilde{\gamma}_{1} / m, \gamma_{2}=\tilde{\gamma}_{2} / m, f=\tilde{f} / m$

Two cases are considered: pure cubic and quadratic-cubic nonlinearity. The first example has simpler dynamic behavior, so all the transformations were purely analytical to demonstrate how the approaches proposed in Section 2 work. The second model with quadratic-cubic nonlinearity demonstrates more complex behavior. It was studied using the approach proposed in Section 3. These models are simple enough to be good proof-of-concept for the present studies.

\subsection{Example 1}

Parameters of the system are taken as follows:

$m=1, c=1, \beta=0.05, \tilde{\gamma}_{1}=0, \tilde{\gamma}_{2}=0.3, \tilde{f}=0.1, \Omega=1.2$.

Equivalent system of the 1st type for such system is the following:

$$
\left\{\begin{array}{l}
x^{\prime \prime}+\Omega^{2} x=y \\
y^{\prime \prime}+h y^{\prime}+\omega^{2} y+6 \gamma_{2} x\left(x^{\prime}\right)^{2}+3 \gamma_{2} x^{2}\left(y-\Omega^{2} x\right)+\gamma_{2} \Omega^{2} x^{3}=0
\end{array}\right.
$$

Invariant manifold corresponding to forced oscillations can be built for this equivalent system by means of the following PDEs: 


$$
\left\{\begin{array}{l}
u \frac{\partial y}{\partial u}+\left(y-\Omega^{2} u\right) \frac{\partial y}{\partial v}=z \\
u \frac{\partial z}{\partial u}+\left(y-\Omega^{2} u\right) \frac{\partial z}{\partial v}=-\left(h z+\omega^{2} y+6 \gamma u v^{2}+3 \gamma u^{2}\left(y-\Omega^{2} u\right)+\gamma \Omega^{2} u^{3}\right)
\end{array}\right.
$$

where $u=x, v=x^{\prime}$.

The invariant manifold corresponding to forced oscillations is calculated using power series (terms up to 5 th degree were kept):

$$
\begin{aligned}
y= & -0.072224 u^{3}-0.002717 u^{2} v+0.150468 u v^{2}+0.000629 v^{3}-0.002751 u^{5}-7.86463210^{-7} u^{4} v- \\
& -0.007306 u^{3} v^{2}-0.000378 u^{2} v^{3}+0.006466 u v^{4}+0.000053 v^{5} \\
z= & 0.003913 u^{3}-0.650020 u^{2} v-0.008152 u v^{2}+0.150468 v^{3}+0.000197 u^{5}-0.014442 u^{4} v+ \\
& +0.000265 u^{3} v^{2}-0.013890 u^{2} v^{3}-0.000660 u v^{4}+0.006467 v^{5}
\end{aligned}
$$

The surfaces $y(u, v)$ and $z(u, v)$ obtained analytically are plotted on the Figure 7 . Having (34) calculated one can construct functions $W$ and $W_{0}$ according to the formulae (24):

$$
\begin{aligned}
& W_{0}=\left(-0.440000 u+0.050000 v+0.227776 u^{3}-0.002717 u^{2} v+0.150468 u v^{2}+0.000629 v^{3}-\right. \\
& \left.-0.002751 u^{5}-7.86463210^{-7} u^{4} v-0.007306 u^{3} v^{2}-0.000378 u^{2} v^{3}+0.006466 u v^{4}+0.000053 v^{5}\right)^{2}+ \\
& +0.694444\left(-0.440000 v-0.072000 u+0.000302 u^{3}+0.249844 u^{2} v-0.000629 u v^{2}+0.150499 v^{3}+\right. \\
& \left.+0.000060 u^{5}-0.014442 u^{4} v-0.000101 u^{3} v^{2}-0.013909 u^{2} v^{3}-0.000337 u v^{4}+0.006470 v^{5}\right)^{2} \\
& W=W_{0}-0.01
\end{aligned}
$$

Graphical representation of $W$ and $W_{0}$ is given on the Figure 8 .

Condition $W=0$ results in periodic orbits of the system (31) on the phase plane.

They are shown on the Figure 9a.

When expressions (34) are known, Rauscher expansions can be built via formulae (22). Their correctness may be checked in the following way. If values of $t, x$ and $x^{\prime}$ are taken from the results of numerical integration and substituted into the Rauscher expansions $\cos (\Omega t)=C\left(x, x^{\prime}\right), \sin (\Omega t)=S\left(x, x^{\prime}\right)$, these expressions must be satisfied. Such check is illustrated on the Figure 9b. Solid lines correspond to the left-hand side of the Rauscher expansions and dots correspond to the righthand side. Calculations are done for the maximum amplitudes regime on Fig.9a. If the system (32) is investigated semi-analytically by means of harmonic balance approach (Section 2), the results of that study confirm analytical ones. For 
example, on the Figure 7 the surfaces $y(u, v)$ and $z(u, v)$ obtained semianalytically are shown (as concentric lines). When plotted together with analytically obtained results, these graphs fit well.

Graphical presentation of $W$ and $W_{0}$ obtained semi-analytically section-wise is given on the Figure 10.

\subsection{Example 2}

As the second example a Duffing equation with nonlinear restoring force of more general type (quadratic-cubic nonlinearity) is considered. Parameters of the system are taken as follows:

$m=1, c=1, \beta=0.02, \tilde{\gamma}_{1}=0.35, \tilde{\gamma}_{2}=0.04, \tilde{f}=0.1, \Omega=0.8$. This system is studied via harmonic balance approach as described in Section 3.

Even for such simple with quadratic-cubic nonlinearity it is rather hard to predict the exact number of periodic solutions for a given value of excitation frequency. This example shows how it can be done using the approach described in Section 3.

First, equations of harmonic balance method (27) were formulated for the equation (35). Continuation parameter was considered to be averaged total energy per period. Then these algebraic equations were differentiated with respect to $\lambda$ and thus transformed into differential ones where $\lambda$ was independent variable and harmonic balance coefficients $A_{0} \ldots A_{n}, B_{2} \ldots B_{n}$ - unknown functions.

These relations were integrated numerically starting from zero values (all variables) to certain value of energy $\lambda^{*}$ which was chosen arbitrary but high enough for finding as many regimes as possible.

For each value of $\lambda$ corresponding value of $\bar{w}(\lambda)$ was calculated via substitution (25) into (24) and subsequent averaging. Whenever $\bar{w}(\lambda)$ became zero, corresponding set of coefficients $A_{0} \ldots A_{n}, B_{2} \ldots B_{n}$ allowed to build periodic orbit of the initial system and its periodic solution (as described in Subsection 3.2). Since the results depend on the number of harmonics taken, the whole calculations were repeated several times while increasing the number of harmonics. On the 
Figure 11 the dependency $\bar{w}(\lambda)$ is shown for different number of harmonics and convergence with respect to the number of harmonics can be clearly observed. The above $a$ priori calculation predicts 5 periodical solutions for the given parameter set. This is confirmed when one constructs amplitude-frequency response of the system (Fig. 12a). Thus, the proposed approach can be used for finding forced periodical response of the system without building its amplitudefrequency dependencies.

Corresponding periodic orbits are depicted on Figure 12b (circles) and compared with the results of numerical simulation (lines).

On Figure 13 the surfaces $y(u, v)$ and $z(u, v)$ are shown.

Surface $W$ obtained section-wise is shown on Figure 14. The results on Fig. 14a are obtained without averaging values for each section. The results on Fig. 14a are obtained after averaging. The sections with too much 'waviness' on Fig. 14a indicate that the number of harmonics taken into account may become insufficient if one desires to investigate regimes with higher amplitudes.

\section{Conclusions}

Rauscher method becomes the matter of interest because in combination with NNM method it allows to calculate steady forced vibrations in multi-DOF mechanical systems via reduction of the number of DOFs. However modern realizations of that approach have significant drawbacks such as iterative nature and the need to have initial approximation for the solution.

The present paper is the first stage on the way of creation similar but non-iterative approach. Currently a new non-iterative variant of Rauscher method is developed for 1-DOF nonlinear dissipative system having analytical nonlinearity.

It is shown that three different types of equivalent autonomous dynamical systems can be built for a given 1-DOF non-autonomous one. The system of $1^{\text {st }}$ type is a $4^{\text {th }}$ order dynamical system. Technically it can be considered as a 2-DOF system where additional "DOF" is explicitly "responsible" for forced oscillations. This system has wider set of periodical orbits than the initial system. The system of $2^{\text {nd }}$ type is a $3^{\text {rd }}$ order dynamical system whose periodic orbits are exactly the same as in the initial system. Using the invariant manifold of the system of $1^{\text {st }}$ type the system of $2^{\text {nd }}$ type can be reduced to the form $W\left(x, x^{\prime}\right)=0$ (which is called the 
equivalent system of the $3^{\text {rd }}$ type). The latter is the most remarkable one. It provides only periodic orbits of the initial system and can be clearly represented geometrically: if one plots the surface $W\left(x, x^{\prime}\right)$, its zero levels become periodic orbits of the initial system. Once $W\left(x, x^{\prime}\right)$ is found: (i) one can obtain different periodical orbits which correspond to forced oscillations in the initial system; (ii) one can estimate amplitudes of vibrations for these regimes; (iii) one can track bifurcations of periodical solutions of the initial system with respect to change of amplitude of external excitation $f$. It is important that the function $W\left(x, x^{\prime}\right)$ can be built a priori via finding the invariant manifold of the equivalent system of $1^{\text {st }}$ type. The same is correct for the Rauscher expansions that can be constructed in the form $\cos (\Omega t)=C\left(x, x^{\prime}\right), \sin (\Omega t)=S\left(x, x^{\prime}\right)$.

As shown in the paper, periodical orbits of the initial non-autonomous system can be obtained via two different approaches: (i) as set of points on phase plane satisfying the condition $W\left(x, x^{\prime}\right)=0$; (ii) via the application of harmonic balance method to the equivalent system of $1^{\text {st }}$ type using system's energy level as a continuation parameter. This approach has advantage over application of harmonic balance method directly to initial system because the latter requires good initial guess for expansion coefficients while the new approach does not. Despite the domain of applicability of the proposed approaches is the matter of further investigations, the results of the current studies are promising, especially if this approach is generalized for multi-DOF systems.

\section{References}

1. Rauscher M. Steady oscillations of system with nonlinear and unsymmetrical elasticity, Journal of Applied Mechanics, 1938, Vol. 5.

2. Kinney W., Rosenberg R.M. On the steady state vibrations of nonlinear systems with many degrees of freedom, J. Appl. Mech, 1966, Vol. 33(2), p. 406-412.

3. Manevich L., Mikhlin Yu. and Pilipchuk V. The method of normal oscillation for essentially nonlinear systems (in Russian), Moscow: Nauka, 1989.

4. Vakakis A., Manevitch L., Mikhlin Yu., Pilipchuk V. and Zevin A. Normal Modes and Localization in Nonlinear Systems, New York: Wiley, 1996.

5. Mikhlin $\mathrm{Yu}$. Resonance modes of near-conservative nonlinear systems. Prikladnaja Matematica I Mekhanica (PMM USSR), 1974, vol. 38, pp.425-429.

6. Avramov K.V. Analysis of forced vibrations by nonlinear modes, Nonlinear Dynamics, 2008, Vol. 53, pp.117-127. 
7. Avramov K.V. Application of nonlinear normal modes to analysis of forced vibrations (in Russian), Applied Mechanics, 2008, № 11, pp.27-33.

8. Mikhlin Y. V., Morgunov B.I. Normal vibrations in near-conservative self-excited and viscoelastic nonlinear systems. Nonlinear dynamics, 2001, vol. 25(1-3), pp. 33-48.

9. Shaw S.W., Pierre C. Non-linear normal modes and invariant manifolds, Journal of Sound and Vibration, 1991, vol.150(1), p.170-173.

10. Shaw S.W., Pierre C. Normal modes for nonlinear vibratory systems, Journal of Sound and Vibration, 1993, Vol. 164, p. 85-124.

11. Avramov K.V., Mikhlin, Yu.V. Nonlinear dynamics of elastic systems (in Russian), Moscow - Izshevsk: NIZ "Regular and chaotic dynamics”, 2010, vol.1, 704 p.

12. Mikhlin, Yu.V., Avramov K.V. Nonlinears Normal Modes for Vibrating Mechanical Systems. Review of Theoretical Developments, Applied Mechanics Reviews, 2010, Vol.63(6), p.060802

13. Perepelkin N.V., Mikhlin Yu.V. and Pierre C. Nonlinear normal forced vibration modes in systems with internal resonance, International Journal of Nonlinear Mechanics, 2013, vol. 57, pp. 102-115.

14. Mikhlin, Y.V., Perepelkin N. V. Nonlinear normal modes and their applications in mechanical systems, Proceedings of the Institution of Mechanical Engineers, Part C: Journal of Mechanical Engineering Science, 2011, 0954406211411254.

15. Perepelkin N.V., Mikhlin, Yu.V. Analysis of forced oscillation modes of a single-disk rotor with nonlinear supports (in Russian), Rigid Body Mechanics, 2010, №40, pp.221232.

16. Perepelkin N.V., Mikhlin Yu.V. Application of nonlinear normal modes theory to the analysis of stationary motions in a model of a single-disk rotor (in Russian), Proceedings of Dniepropetrovsk National University. Series: Methods of solution of applied problems of mechanics of deformable bodies, 2012, vol.13, pp. 327-336

17. Avramov K.V., Mikhlin, Yu.V. Nonlinear dynamics of elastic systems (in Russian), Moscow - Izshevsk: Institute of computer researches, 2015, vol.2, 700 p.

18. Klimenko A.A., Mikhlin Y.V. and Awrejcewicz J. Nonlinear normal modes in pendulum systems, Nonlinear Dynamics, 2012, vol. 70(1), pp. 797-813.

19. Uspenskiy B.V., Avramov K.V. Nonlinear normal modes of piecewise systems (in Russian), Bulletin of V.N. Karazin Kharkiv National University, 2012, vol. 1018, pp.7683.

20. Pesheck E., Pierre C. and Shaw S.W. A new Galerkin - based approach for accurate non-linear normal modes through invariant manifolds, Journal of Sound and Vibration, 2002,Vol. 249, No 5, pp. 971-993. 


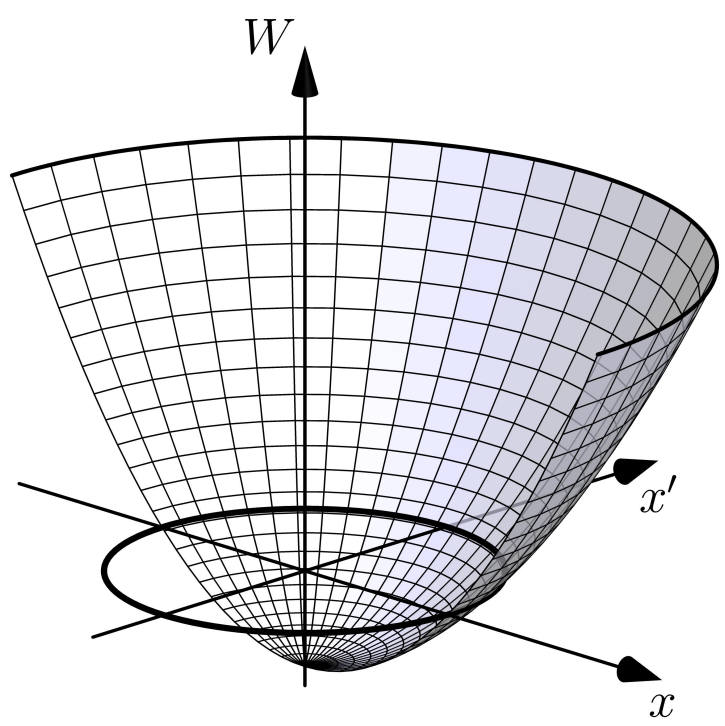

Figure 1. The W surface

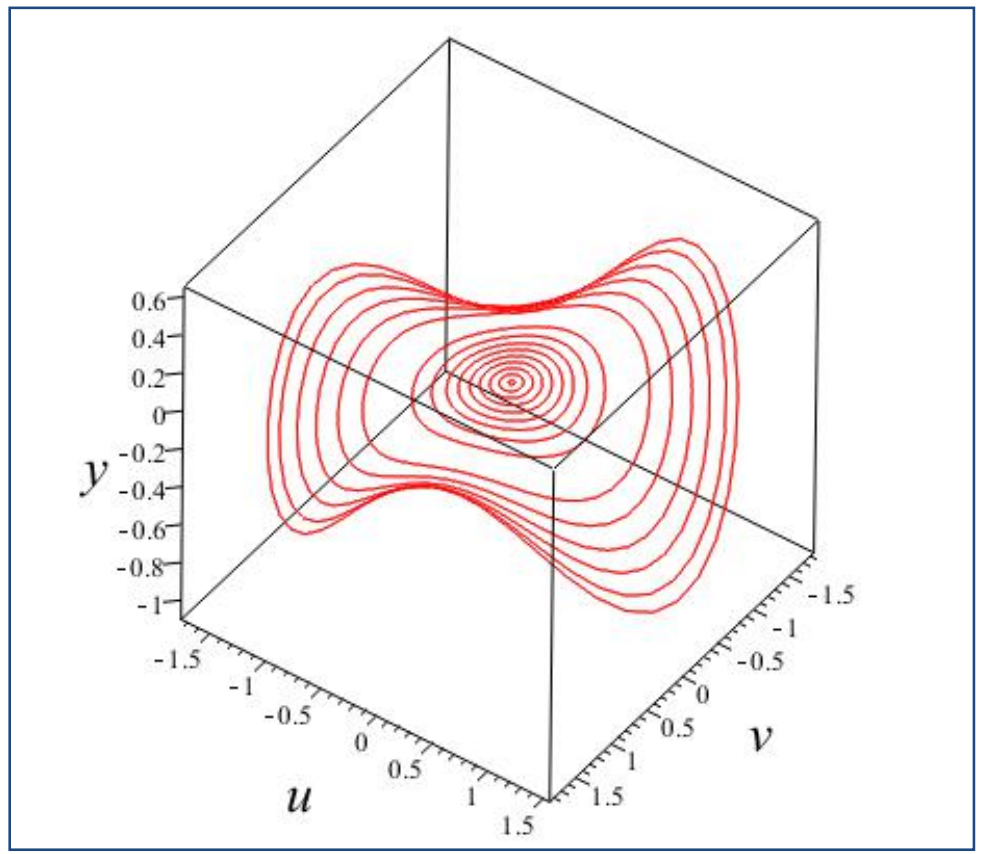

Figure 2. Invariant manifold of the system (18) is fully covered with periodic orbits of the initial system. 


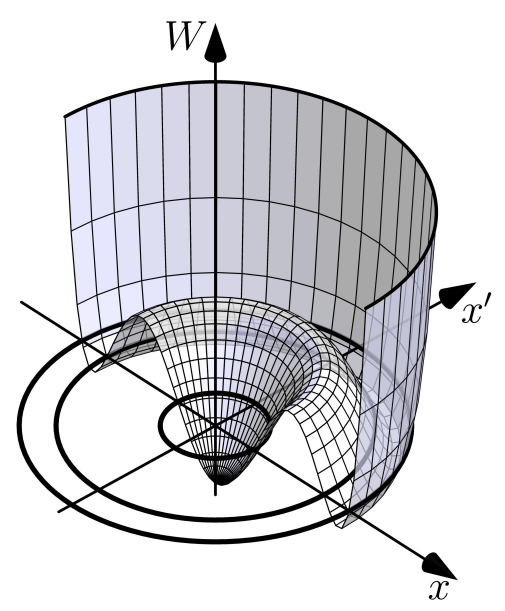

(a)

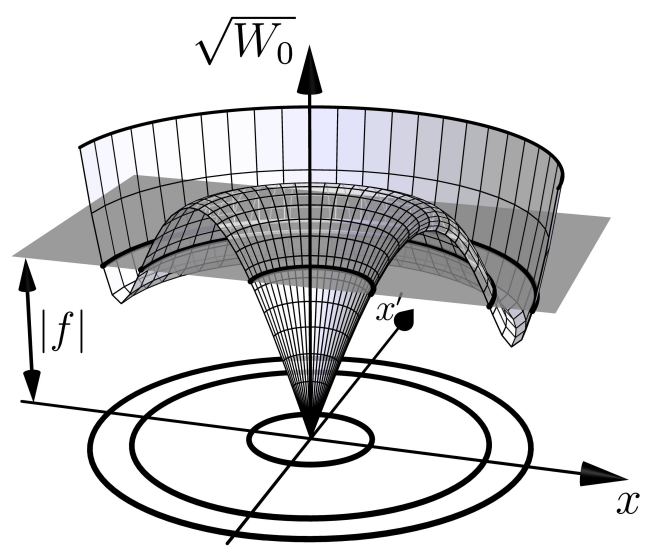

(b)

Figure 3. Geometrical interpretation of surfaces $\mathrm{W}-(\mathrm{a})$ and $\mathrm{W}_{0}-(\mathrm{b})$.

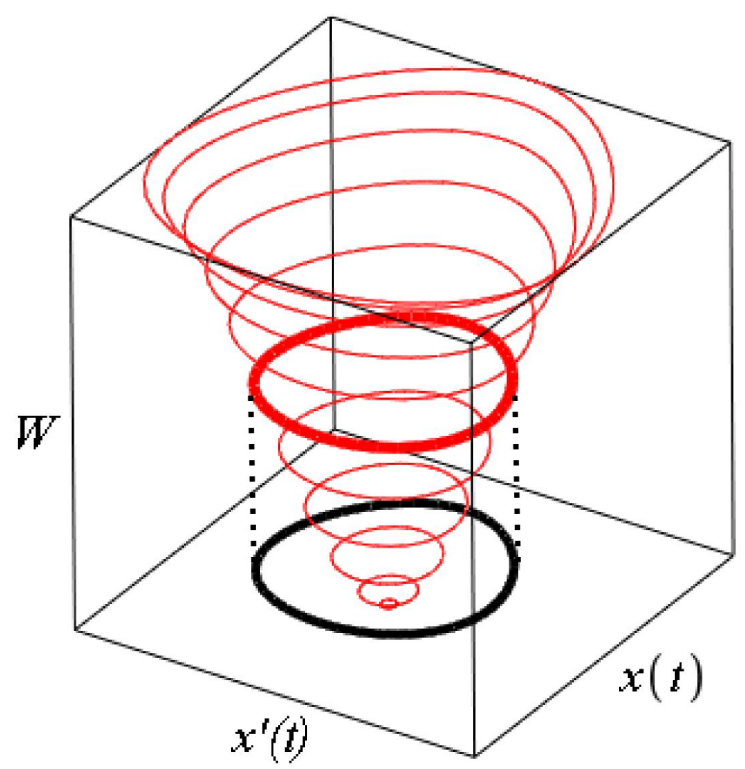

Figure 4. Level curves of the W surface. 


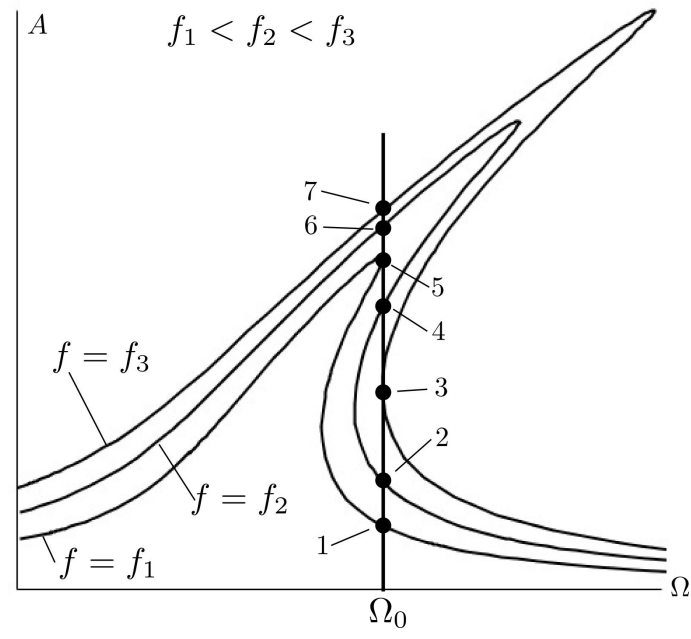

(a)

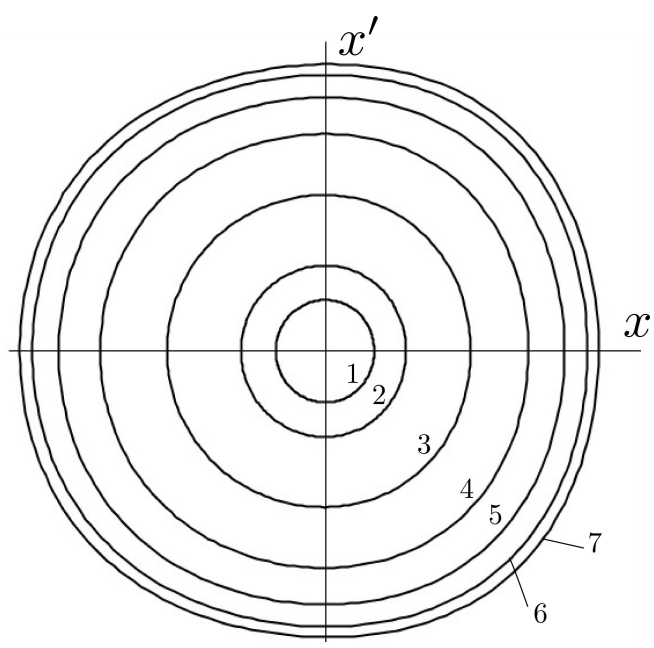

(b)

Figure 5. Amplitude-frequency responses - (a) and different periodic orbits corresponding to frequency value $\Omega=\Omega_{0}$ - (b)

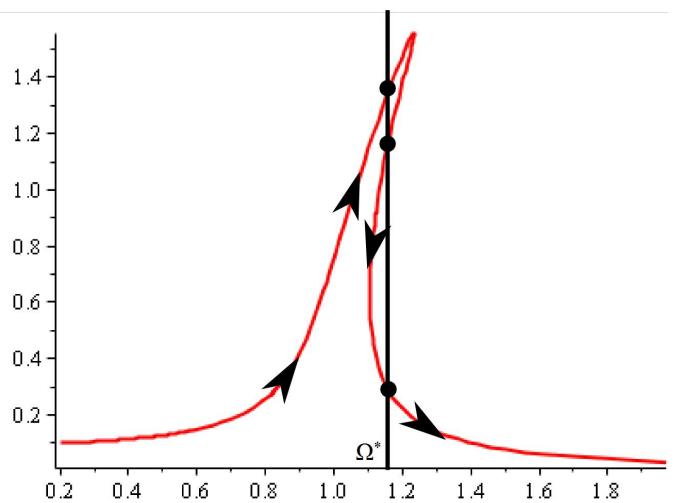

(a)

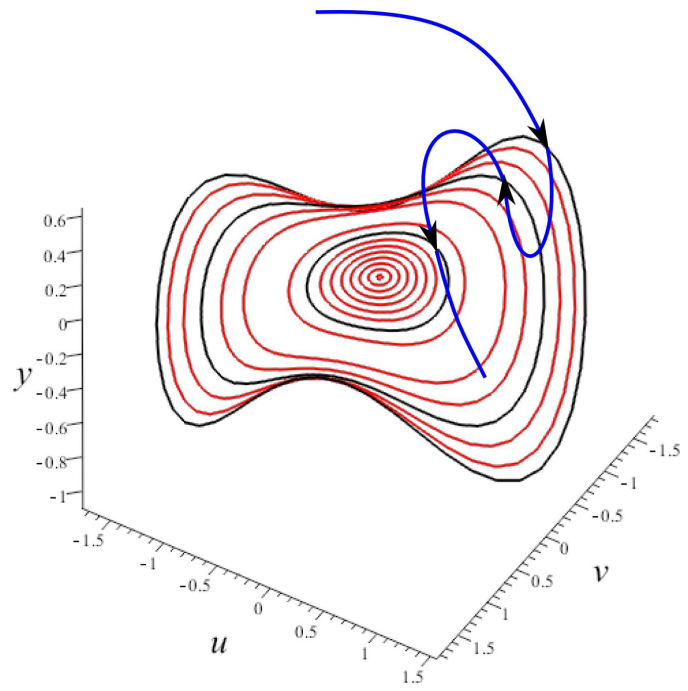

(c)

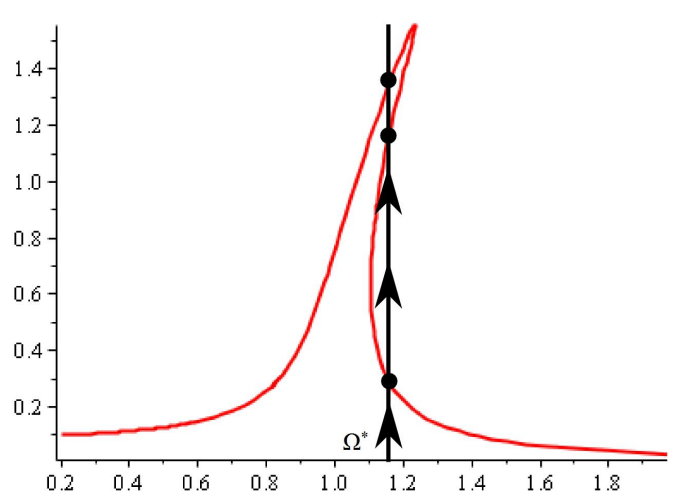

(b)

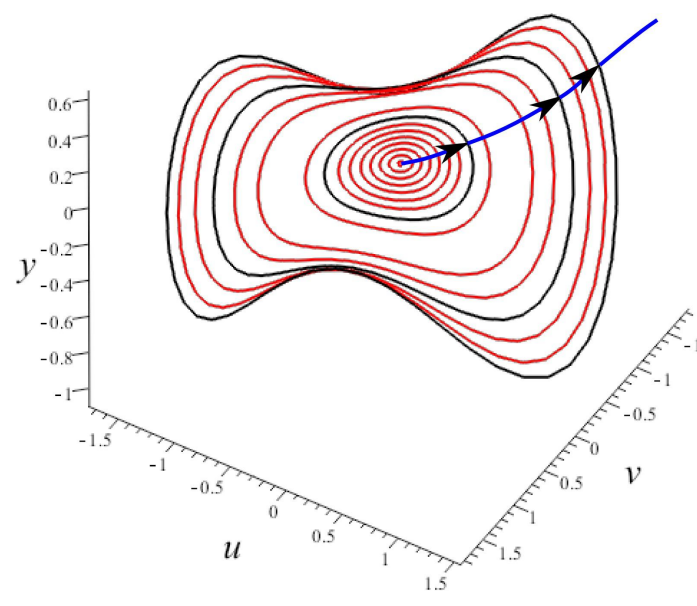

(d) 
Figure 6. Continuation after application of harmonic balance method to initial $(\mathrm{a}, \mathrm{c})$ and equivalent (b,d) systems.

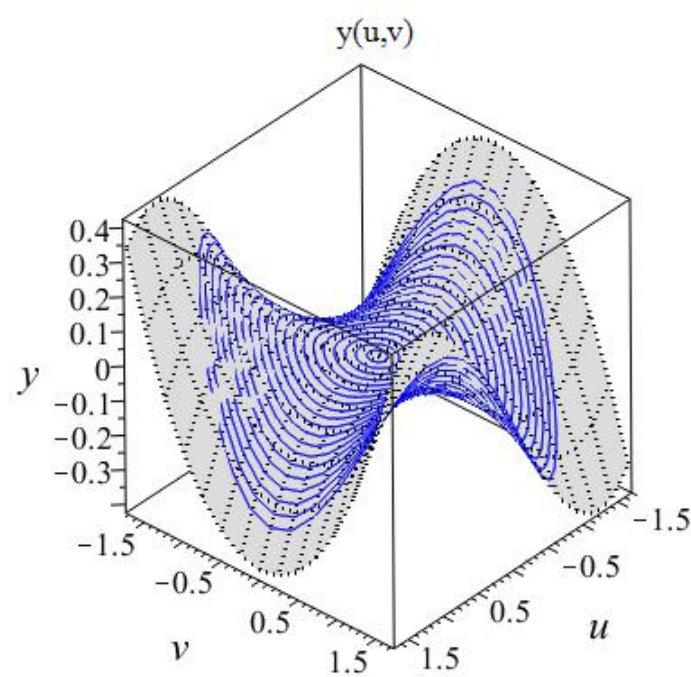

(a)

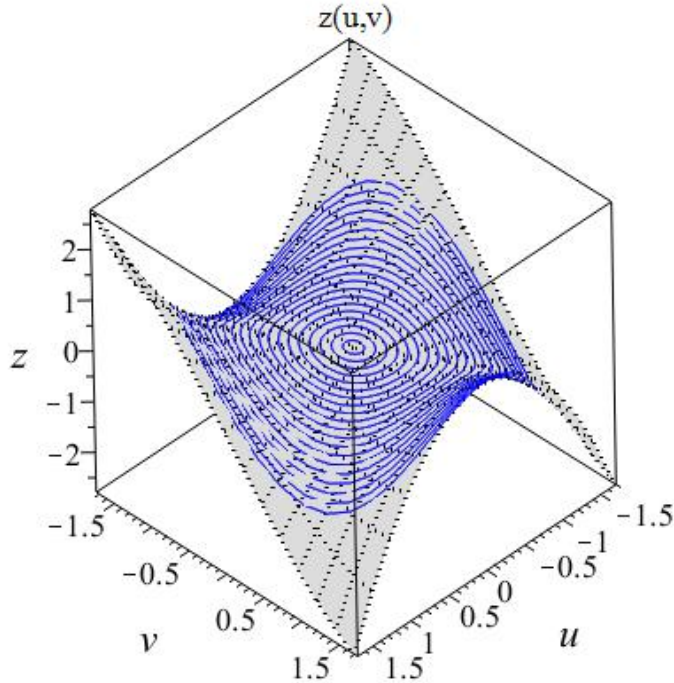

(b)

Figure 7. Comparison of the invariant manifold surfaces of the equivalent system of the 1st type (32) obtained analytically (grey surfaces) and semi-analytically (concentric lines): $y(u, v)$ - (a), $z(u, v)-(\mathrm{b})$

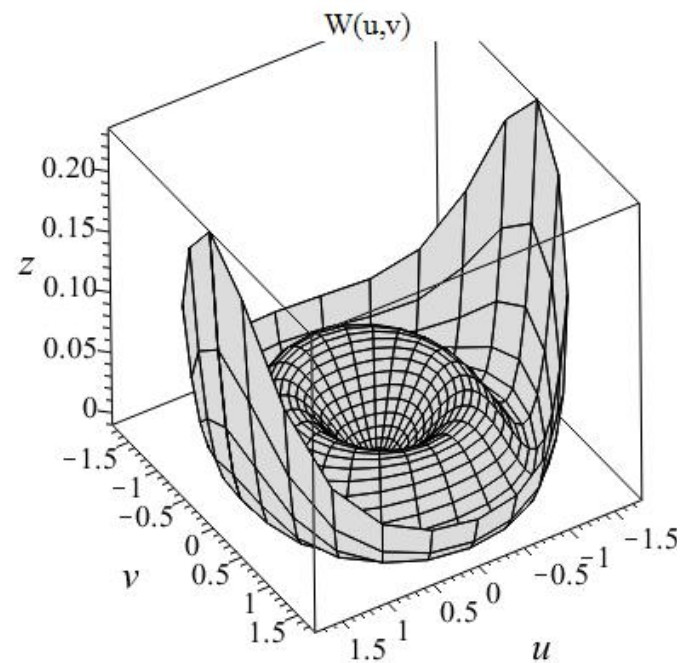

(a)

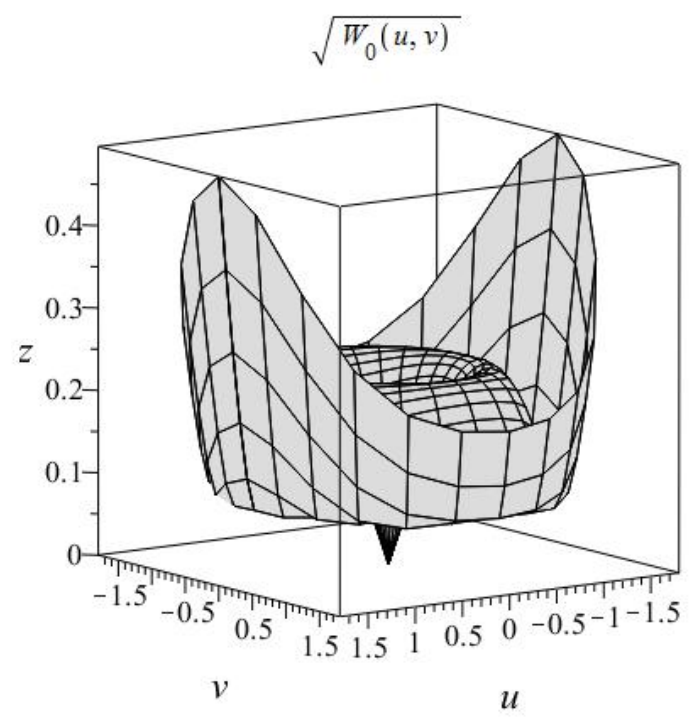

(b)

Figure 8 . Graphical representation of $\mathrm{W}-(\mathrm{a})$ and $\mathrm{W}_{0}-(\mathrm{b})$ obtained analytically 


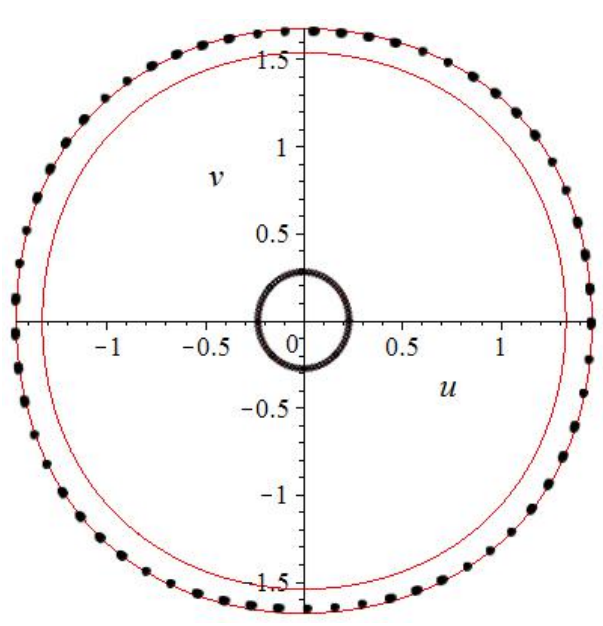

(a)

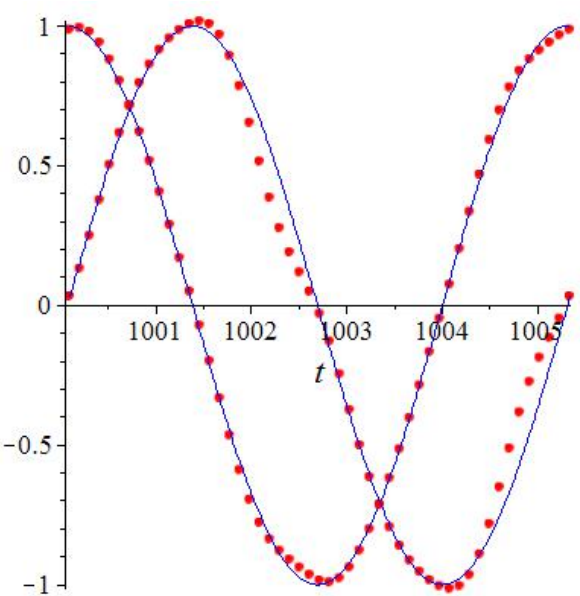

(b)

Figure 9. (a) - Periodical orbits obtained analytically (thin lines) are compared with the results of numerical integration (dots); (b) - Rauscher expansions quality check

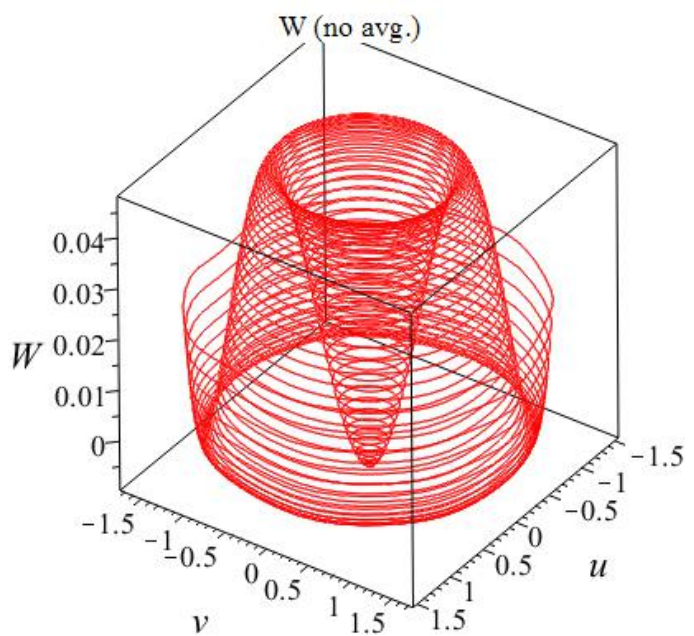

(a)

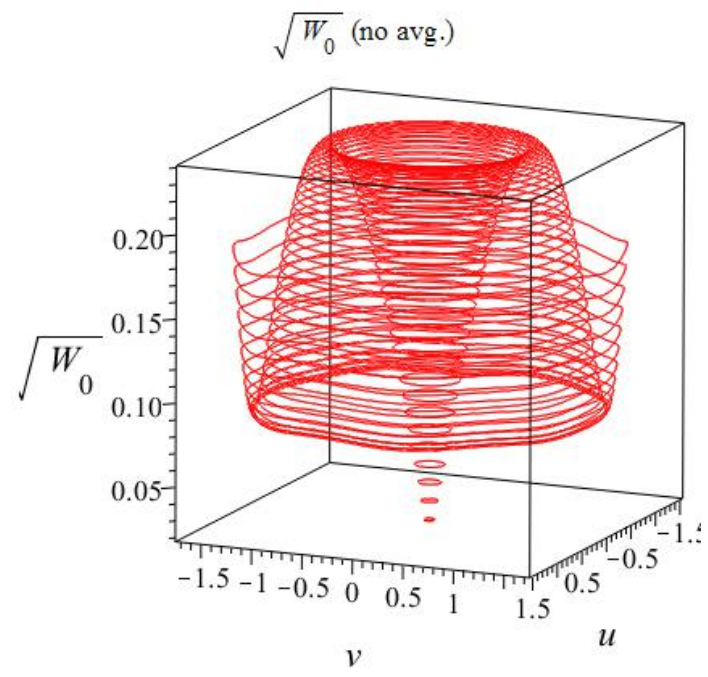

(b)

Figure 10. Graphical representation of $\mathrm{W}-(\mathrm{a})$ and $\mathrm{W}_{0}-(\mathrm{b})$ obtained semi-analytically. 


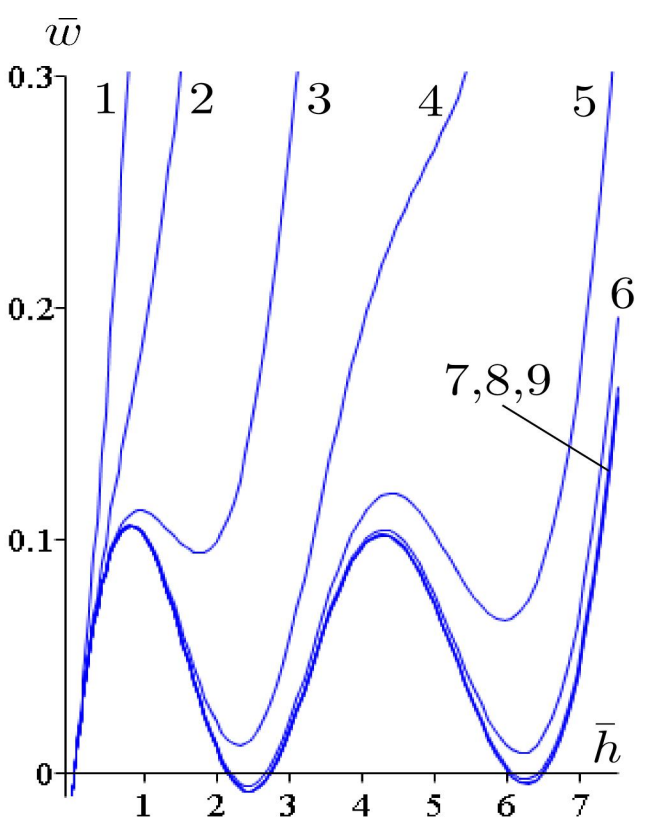

Figure 11. The dependencies $\bar{w}(\lambda)$ obtained for the case $\lambda$ is the averaged total energy $\bar{h}$. Numbers show the number of harmonics used.

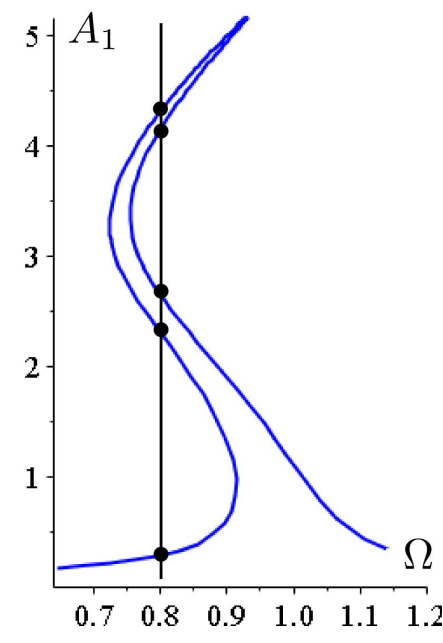

(a)

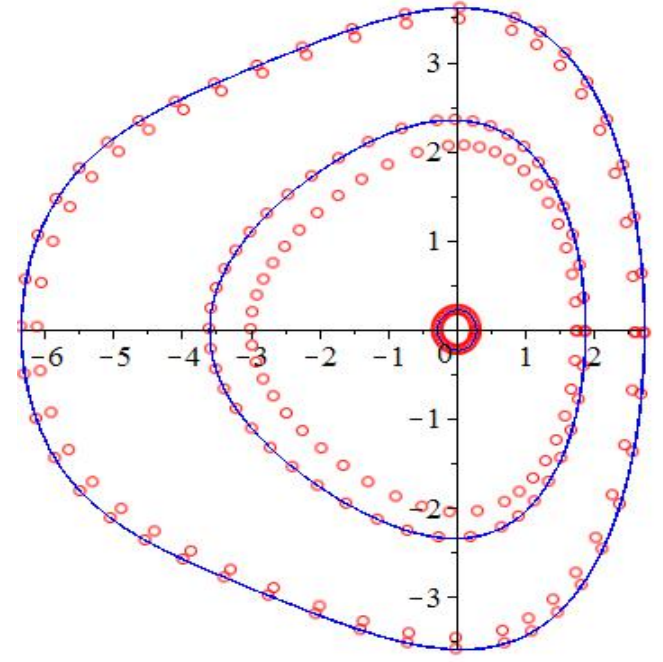

(b)

Figure 12. Amplitude-frequency response of the system (1st harmonic) - (a); closed trajectories corresponding to $\Omega=0.8$ - (b) 


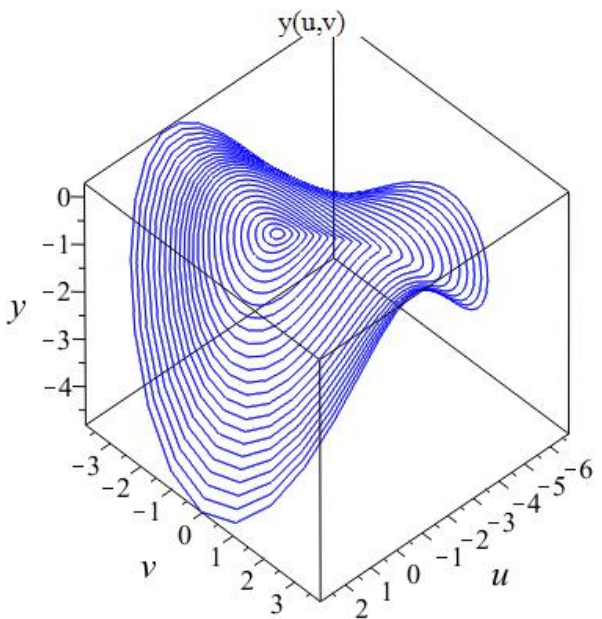

(a)

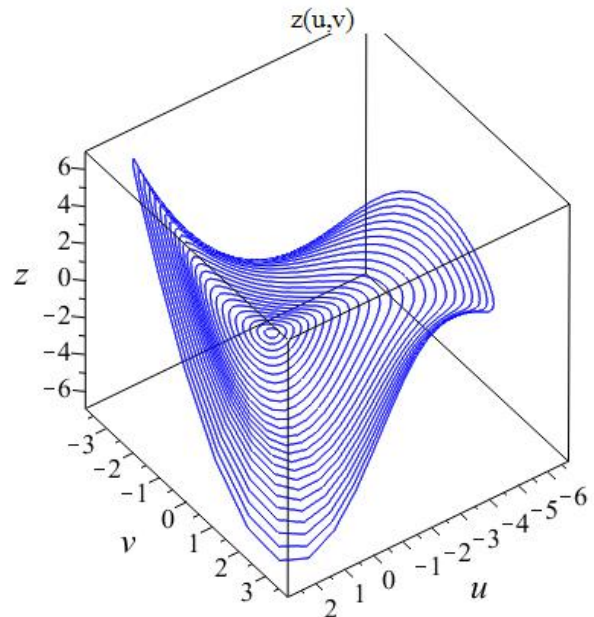

(b)

Figure 13. Dependencies $y(u, v)$ - (a), $z(u, v)$ - (b) obtained semi-analytically.

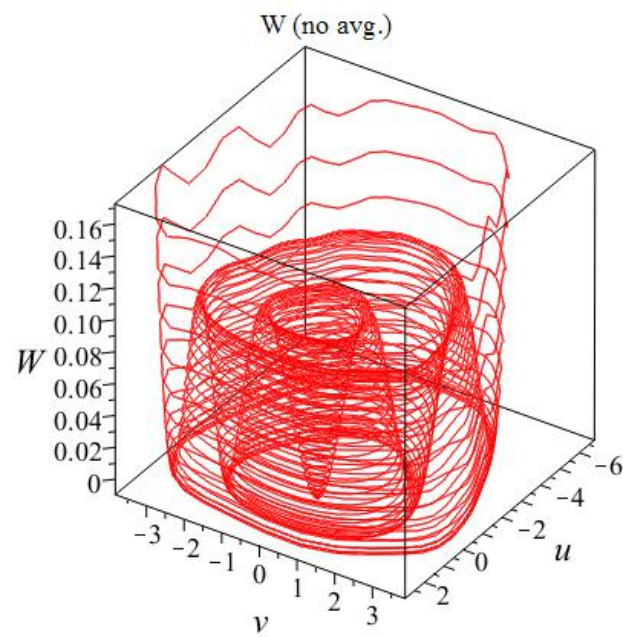

(a)

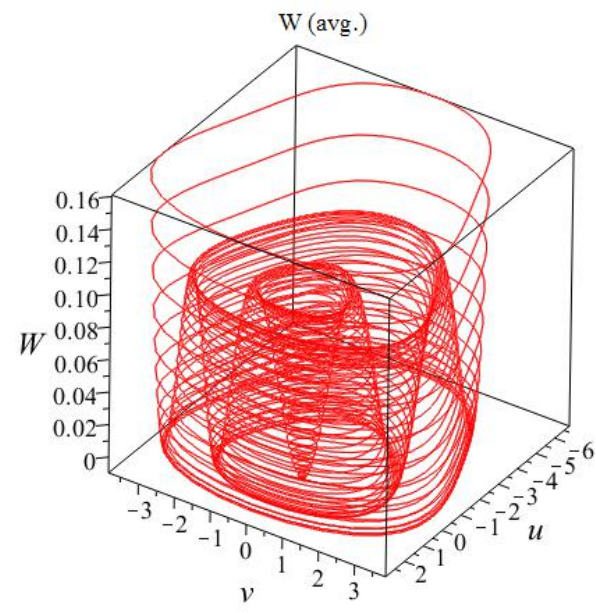

(b)

Figure 14. Sections of the $W$ surface obtained without (a) and with (b) averaging the results. 